\title{
A assistência suplementar de saúde e seus projetos de cuidado para com o idoso
}

\author{
Elderly care in the supplementary health system
}

Renato Peixoto Veras ${ }^{1}$

Célia Pereira Caldas ${ }^{1}$

Denizar Viana Araújo ${ }^{2}$

Rosana KuschniereWalter M endes ${ }^{3}$

${ }^{1}$ UniversidadeA berta da Terceiraldade,

Universidade do Estado do

Rio deJaneiro. Rua São

Francisco Xavier 524/Bloco

$\mathrm{F} / 10.150, \mathrm{M}$ aracanã.

20540-900 Rio deJaneiro

RJ.veras@uerj.br

${ }^{2}$ Departamento de

Economia da Saúde.

UniversidadeFederal deSão

Paulo.

${ }^{3}$ Escola N acional de Saúde

Pública, Fundação O swaldo

Cruz.
Abstract The purpose of this study was to identify and analyze the assistance provided to the elderly by the supplementary health care system. Information was collected from interviews with managers of chronic diseases management programs and/or of specific programs in this area. Information wasorganized in casesand analyzed. Eleven companies operating private health plans in the cities of Rio de Janeiro and Sao Paulo were investigated. A general care model for the elderly was defined for the analysis. This model consists in a proposal for a care and assistance system hierarchically organized according to the deterioration of the functional capacity of the elderly (levels of dependence). N one of the six companies that had health plans for the elderly already implemented provided full assistance to all enrolled seniors. There are major differences in the stage of development of their programs. Four companies had al ready implemented plansand two companies had plans in development. Even with the teams of managers and professionals working on some level within the advocated model, none of these companies followed any logic of integrated care and monitoring in all stages of the ageing process in accordance with the degree of dependence of the associate.

Key words Elderly, Supplementary health care system
Resumo 0 propósito deste estudo foi identificar e analisar atividades de atenção ao idoso no âmbito do sistema de saúde suplementar a partir de entrevistas com gestores deprogramas de gerenciamento de doenças crônicas e/ou programas específicos para este segmento. As informações coletadas foram organizadas na forma de casos e analisadas. Os dadosse referem a onzeempresas operadoras de planos privados de saúde nas cidades do Rio de Janeiro e São Paulo. Para a análise, foi definido um modelo geral de atenção ao idoso. Este modelo é uma proposta de hierarquização dos cuidados e da assi stência, baseado na deterioração da capacidade funcional do idoso (grau de dependência). Entreas seis empresas que têm planos implantados, nenhuma empresa apresentou pleno atendimento a todos os idosos de suas respectivas carteiras. Há grandes diferenças quanto ao estágio de implantação de seus programas. Quatro empresas têm programas implantados e duas empresas têm programas em implantação. M esmo quando a equipe de gestorese de profissionais atuava dentro de algum nível do modelo preconizado, não foi identificada em empresa alguma uma lógica de cuidado integral emonitoramento em todas as etapas do envelhecimento, visando uma continuidade dasações, de acordo com o grau de dependência do associado.

Palavras-chave Idoso, Sistema de saúde suplementar 
Introdução

Em menos de trinta anos, o Brasil passou de um perfil de morbi-mortalidade típico de uma população jovem para um quadro caracterizado por enfermidades complexas e onerosas, próprias das faixas etárias mais avançadas.

O M inistério da Saúderecentementeincluiu a saúde do idoso como item prioritário na agenda de saúde do país, promulgando uma nova política nacional de saúde da pessoa idosa baseada no paradigma da capacidade funcional, que é abordada de maneira multidimensional ${ }^{1}$. M as 0 efeito prático ainda não foi sentido. 0 peso assistencial ainda é preponderante e o notável grau de desarticulação dentro do sistema de saúde dificulta a operacionalização de qualquer lógica baseada em uma avaliação multidimensional.

Em grande parte, tais desafios são respondidos pelo Sistema Suplementar de Saúde. No entanto, pouco se sabe da abrangência e do impacto desta resposta. Portanto, faz-se necessário explorar esta nova realidade para que seja possível otimizar, bem como regular, os agentes prestadores de serviços e suas ações? ${ }^{2}$.

0 propósito desteestudo foi identificar eanalisar práticas eatividades de aten ção ao idoso no âmbito do sistema de saúde suplementar a partir de entrevistas com gestores de programas de gerenciamento de doenças crônicas e/ou programas específicos para este segmento, em operadoras de planos de saúde. A intenção é contribuir para a discussão de políticas/estratégias que permitam a indução de modelos de atenção ao idoso com ênfase em projetos preventivos e resolutivos, visando a detecção precoce de agravos à saúde e que incorporem metodologias de avaliação ampla em Geriatria, permitindo gestão mais eficiente do cuidado ao idoso fragilizado e com múltiplas patologias.

Para isso, foram coletadas informações junto a onze empresas operadoras de planos privados de saúde nas cidades do Rio de J aneiro e São Paulo. Algumas empresas desenvolvem ações específicas para estas cidades eoutras mantêm ações em âmbito nacional. 0 estudo utilizou uma abordagem qualitativa buscando descrever e analisar cada modelo assistencial.

\section{M etodologia}

Trata-se de um estudo qualitativo fundamentado teoricamente nos campo da saúde coletiva e da gerontologia e nas áreas do planejamento e gestão em saúde. Foram levantadas informações relativas aos serviços, programas e atividades alternativas dirigidos especificamente para os idosos, no sistema de saúde suplementar. Estas informações foram organizadas na forma de casos e analisadas.

Foram selecionadas onze empresas operadoras de planos privados de saúde nas cidades do Rio de Janeiro e São Paulo. 0 primeiro critério para sel eção destas operadoras foi a sua relevância no mercado, considerando o número devidas cobertase experiência no gerenciamento dedoenças. U ma vez identificadas as grandes empresas, buscou-se selecionar representantes de cada tipo de operadora. D efiniu-se então que seriam estudadas quatro empresas de autogestão (Empresas A, B, C, D ); quatro empresas de M edicina de Grupo (Empresas E, F, G, H) ; duas empresas seguradoras especializadas em saúde (Empresas I, J) e uma cooperativa médica (Empresa K).

Optou-se por não incluir na amostra empresa do tipo filantrópica, por não ter sido encontrada empresa filantrópica que atendesse ao primeiro critério de inclusão, ou seja, a relevância no mercado.

O Rio de Janeiro e São Paulo possuem um grande contingente de idosos associados a grandes empresas prestadoras de saúde suplementar. Portanto, optou-se por selecionar as empresas situadas nestas cidades.

Os informantes são gerentes e funcionários do escalão superior das empresas, envolvidos direta ou indiretamentena formulação de políticas de gerenciamento de crônicos da empresa. Deve ser ressaltado que houve total receptividadena maioria das empresas. D os onze casos analisados, apenas três empresas não se mostraram receptivas (EmpresasI, J eF). Assim, foi decidido descrever estas empresas tendo como fonte material de divulgação de seus programas, considerando serem de domínio público. N os três casos citados, portanto, não há informações sobre indicadores de estrutura, processo e de resultado. Por outro lado, entre as empresas que optaram por participar do estudo, os resultados têm uma "qualidade superior" a muitas descrições disponíveis na literatura sobre o funcionamento dos serviços do setor de saúde suplementar.

Os dados foram colhidos através da técnica de entrevista semi-estruturada. Um questionário foi utilizado como um roteiro. Este questionário foi construído a partir do enfoque para avaliação de serviços de saúde propostos por Donabedian ${ }^{3-5}$, a saber: avaliação de estrutura, de processo e de resultado/impacto. 
Por ser um roteiro, no momento da aplicação, nem todas as perguntas se enquadravam no perfil da empresa e, portanto, foram omitidas durante a entrevista. Este fato propiciou que alguns pontos fossem mais aprofundados em algumas empresas do que em outras. No entanto, ao adotar o roteiro, foi garantido que os pontos definidos como chaves fossem abordados.

Foi realizado pré testedo instrumento decoleta de dados. Esta etapa foi realizada na Empresa $\mathrm{E}$, com a presença todos os pesquisadores. $O$ entrevistado foi o gerente sênior de formulação de políticas de gerenciamento de crônicos da empresa.

Para a análise dos dados, foi definido um modelo geral de atenção ao idoso. Este modelo é uma proposta de hierarquização dos cuidados e da assistência, baseado na deterioração da capacidade funcional do idoso (grau de dependência). De acordo com este modelo, que tem quatro níveis, parte-se do pressuposto que o maior fator de risco é a idade. Portanto, todos os idosos devem ser contemplados com algum tipo de cuidado, desde o mais leve, apenas para têlo sob acompanhamento, atéaqueles que necessitam de intervenção de grande monta. 0 tipo de ação a ser oferecida será definido pelo grau de dependência. N estemodelo, a internação deveser considerada uma intervenção para casos agudos e, uma vez resolvida a situação de risco, os cuidados devem ter sua continuidade na residência do cliente, com uma abordagem domiciliar, seja assistência ou internação domiciliar.

0 modelo teórico apresentado no Q uadro 1 é explicativo, embasado teoricamente no campo da saúde coletiva e nos concei tos que envolvem a noção de envelhecimento saudável. Na Organização M undial de Saúde, este campo se organiza em torno de políticas do envel hecimento ativo ${ }^{6}$. Neste modelo, a internação hospitalar não está colocada. 0 objetivo do modelo é procurar entender os níveis de dependência eatenção ao idoso fora do período de internação.

0 modelo não é conhecido pelos planejadores das operadoras de saúde e pelos profissionais de saúde que militam na área da assistência ao idoso. Ele representa um acúmulo de conhe cimentos e práticas de estudiosos que trabalham em torno da Universidade Aberta da Terceira Idade (UnATI) - um programa de pesquisa e atenção integral à saúde do idoso, desenvolvido na Universidade do Estado do Rio de Janeiro. Elefoi elaborado para ser utilizado inicialmente como marco conceitual deste estudo. Este modelo orientou o questionário e as entrevistas realizadas neste trabalho.

\section{Resultados}

Entre as onze empresas selecionadas para o estudo, três se recusaram a participar. Ainda assim, foram trabalhados dados obtidos de material de divulgação. No entanto, tais casos foram considerados inconsistentes para análise.

Entre as oito empresas cujos dados apresentam consistência para análise, duas não têm programas com ações específicas para o segmento de idosos - a Empresa B e a Empresa C, a qual atua com a abordagem de saúde da família. Dentre as que possuem programa para idosos, quatro empresas implementam os seus próprios programas (Empresa E, Empresa A, Empresa G e Empresa D) eduas empresas terceirizam o serviço. N estas empresas, cujo serviço prestado se dá através de outras empresas terceirizadas, há diferenças quanto ao grau de autonomia das terceiras. $\mathrm{Na}$ Empresa K, 0 acompanhamento das ações é feito por monitoramento e auditoria. $\mathrm{Na}$ Empresa $\mathrm{H}$, há uma enfermeira da empresa que gerencia cada caso, através de relatórios mensais das empresas tercei rizadas e visitas al eatórias aos beneficiários submetidos ao programa.

O Q uadro 2 ilustra o panorama encontrado nestas empresas. Entre as seis empresas que têm planos implantados, nenhuma apresentou pleno atendimento a todos os idosos de suas respectivas carteiras. No entanto, há grandes diferenças quanto ao estágio de implantação de seus programas. Quatro empresas (Empresa H, Empresa $G$, Empresa $K$ e Empresa A) têm programas implantados e duas empresas (Empresa D e Empresa E) têm programas em implantação. M esmo entre as que têm programas implantados, pode ser observado que o percentual deidosos atendidos é mínimo em relação aos el egíveis em suas carteiras.

De acordo com o modelo exposto no Quadro 1, as Empresas K, G e D foram consideradas as quemelhor atendem as necessidades destesegmento. As Empresas A, C, E, e H desenvolvem ações em al gum nível, compatíveis com o modelo. A Empresa B não desenvolve qualquer ação preconizada pelo modelo. As Empresas J, F e I não concederam entrevistas e não puderam ser adequadamente avaliadas.

Um resultado contundente foi a constatação que mesmo as empresas que apresentam programas mais completos, de acordo com o modelo, atuam num universo extremamente limitado de indivíduos. 
Quadro 1. M odelo teórico baseado na deterioração da capacidade funcional do idoso (grau de dependência).

\begin{tabular}{|c|c|c|c|c|}
\hline $\begin{array}{c}\text { Nível de } \\
\text { complexidade }\end{array}$ & nível 1 & nível 2 & nível 3 & nível 4 \\
\hline $\begin{array}{l}\text { Estimativa da } \\
\text { proporção da } \\
\text { clientela }\end{array}$ & $\begin{array}{l}\text { Em torno de } 55 \% \\
\text { de clientes }\end{array}$ & $\begin{array}{l}\text { Em torno de } 25 \% \\
\text { de clientes }\end{array}$ & $\begin{array}{l}\text { Em torno de } 15 \% \\
\text { de clientes }\end{array}$ & $\begin{array}{l}\text { Em torno de } 5 \% \\
\text { de clientes }\end{array}$ \\
\hline $\begin{array}{l}\text { Grau de } \\
\text { dependência }\end{array}$ & Saudável e independente & Dependência leve & Dependência moderada & $\begin{array}{l}\text { Fragilizado e } \\
\text { dependente }\end{array}$ \\
\hline $\begin{array}{l}\text { Ações } \\
\text { propostas }\end{array}$ & $\begin{array}{l}\text { - Promoção da saúde } \\
\text { - Educação em saúde } \\
\text { - Atenção Básica à Saúde } \\
\text { - Monitoramento da } \\
\text { saúde e detecção de risco } \\
\text { de fragilização }\end{array}$ & $\begin{array}{l}\text { - Educação em saúde } \\
\text { - Assistência } \\
\text { ambulatorial de baixa } \\
\text { complexidade } \\
\text { - Reabilitação } \\
\text { - Visita domiciliar } \\
\text { - Gerenciamento de } \\
\text { caso }\end{array}$ & $\begin{array}{l}\text { - Assistência } \\
\text { ambulatorial ou } \\
\text { domiciliar de média } \\
\text { complexidade } \\
\text { - Abordagem } \\
\text { gerontológica } \\
\text { - Reabilitação } \\
\text { - Assistência Domiciliar } \\
\text { - Gerenciamento de caso } \\
\text { com monitoramento da } \\
\text { fragilidade }\end{array}$ & $\begin{array}{l}\text { - Assistência } \\
\text { ambulatorial ou } \\
\text { domiciliar geriátrica } \\
\text { - Atenção especializada } \\
\text { para suporte nas } \\
\text { atividades de vida diária } \\
\text { - Assistência hospitalar } \\
\text { durante o dia } \\
\text { - Reabilitação } \\
\text { - Assistência domiciliar } \\
\text { planejada por nível de } \\
\text { complexidade } \\
\text { - Apoio aos cuidadores }\end{array}$ \\
\hline $\begin{array}{l}\text { Cenário } \\
\text { das ações }\end{array}$ & $\begin{array}{l}\text { - Centro de convivência } \\
\text { - Consultórios }\end{array}$ & $\begin{array}{l}\text { - Centro de convivência } \\
\text { - Consultórios } \\
\text { - Ambulatório } \\
\text { - Domicílio }\end{array}$ & $\begin{array}{l}\text { - Ambulatório } \\
\text { - Consultórios } \\
\text { - Domicílio } \\
\text { - Centro de reabilitação }\end{array}$ & $\begin{array}{l}\text { - Ambulatório } \\
\text { - Centro-dia } \\
\text { - Hospital-dia } \\
\text { - Centro de reabilitação } \\
\text { - Domicílio }\end{array}$ \\
\hline $\begin{array}{l}\text { Qualificação } \\
\text { necessária } \\
\text { para a equipe } \\
\text { de saúde }\end{array}$ & $\begin{array}{l}\text { Capacitação para ações } \\
\text { de promoção de saúde }\end{array}$ & $\begin{array}{l}\text { Capacitação em } \\
\text { Geriatria e Gerontologia }\end{array}$ & $\begin{array}{l}\text { - Especialização em } \\
\text { Geriatria e Gerontologia } \\
\text { - Curso de cuidadores }\end{array}$ & $\begin{array}{l}\text { Especialização com } \\
\text { formação prática em } \\
\text { Geriatria e Gerontologia } \\
\text { - Curso de cuidadores }\end{array}$ \\
\hline
\end{tabular}

\section{Discussão}

0 modelo teórico que serviu de base para esta discussão (Quadro 1) aponta que as ações requeridas para o cuidado ao idoso devem ser estratificadas em quatro níveis, de acordo com 0 grau de dependência do idoso e com a complexidade das ações. 0 pressuposto básico é que a idade é o maior fator de risco para a dependên$\mathrm{cia}^{7,8}$. N este sentido, a melhor estratégia para um adequado cuidado para com o idoso é, utilizando uma lógica de permanente monitoramento da saúde visando à prevenção de agravos, tê-lo sempre sob observação, variando apenas os níveis, a intensidadee o cenário da intervenção $0^{9,10,11}$.

Este modelo é baseado na preservação da ca- 
Quadro 2. Empresas com programas específicos para o idoso classificados por ações, níveis de atenção oferecidos e resultados.

\begin{tabular}{|c|c|c|c|c|}
\hline $\begin{array}{c}\text { Empresa por } \\
\text { tipo }\end{array}$ & $\begin{array}{l}\text { Vinculação } \\
\text { do programa }\end{array}$ & $\begin{array}{l}\text { Estágio de } \\
\text { implantação }\end{array}$ & Ações & $\begin{array}{c}\text { Níveis de atenção } \\
\text { contemplados }\end{array}$ \\
\hline \multicolumn{5}{|l|}{ Autogestão } \\
\hline Empresa A & Próprio & Completo & $\begin{array}{l}\text { Prevenção de doenças crônicas; estratégia } \\
\text { de saúde da família; assistência domiciliar; } \\
\text { campanhas educativas e de vacinação }\end{array}$ & $\begin{array}{l}\text { - nível } 1 \\
\text { - nível } 2\end{array}$ \\
\hline Empresa D & Próprio & Em processo & $\begin{array}{l}\text { Educação em saúde; Centro de } \\
\text { convivência; assistência domiciliar; } \\
\text { gerenciamento de crônicos; gerenciamento } \\
\text { de risco }\end{array}$ & $\begin{array}{l}\text { - nível } 1 \\
\text { - nível } 2\end{array}$ \\
\hline \multicolumn{5}{|l|}{$\begin{array}{l}\text { Medicina de } \\
\text { grupo }\end{array}$} \\
\hline Empresa G & Próprio & Completo & $\begin{array}{l}\text { Gerenciamento de crônicos; educação em } \\
\text { saúde; visita domiciliar; monitoramento } \\
\text { telefônico; avaliação geriátrica; assistência } \\
\text { domiciliar de acordo com grau de } \\
\text { dependência }\end{array}$ & $\begin{array}{l}\text { - nível } 1 \\
\text { - nível } 2 \\
\text { - nível } 3 \\
\text { - nível } 4 \text { (parcial) }\end{array}$ \\
\hline Empresa H & Terceirizado & Em processo & $\begin{array}{l}\text { Gerenciamento de crônicos; visita } \\
\text { domiciliar; educação em saúde; assistência } \\
\text { domiciliar }\end{array}$ & $\begin{array}{l}\text { - nível } 1 \\
\text { - nível } 2\end{array}$ \\
\hline $\begin{array}{r}\text { Cooperativa } \\
\text { Empresa } \mathrm{K}\end{array}$ & Terceirizado & Completo & $\begin{array}{l}\text { Gerenciamento de crônicos; } \\
\text { monitoramento por telefone; Visita } \\
\text { domiciliar; educação em saúde; assistência e } \\
\text { internação domiciliar }\end{array}$ & $\begin{array}{l}\text { - nível } 1 \\
\text { - nível } 2 \\
\text { - nível } 3 \\
\text { - nível } 4 \text { (parcial) }\end{array}$ \\
\hline
\end{tabular}

pacidade funcional epartedo pressuposto deque todos os idosos devem ser acompanhados, porém em diferentes graus. Portanto, o modelo tem quatro níveis de complexidade, desdeaquelecom ações a serem adotadas para os idosos saudáveis eindependentes, atéo estágio demaior complexidade, que visa idosos fragilizados e dependentes.

$\mathrm{N}$ a série de entrevistas realizadas, observouse que mesmo quando a equipe de gestores e de profissionais atuavam dentro de algum nível do modelo preconizado, não foi identificada em empresa alguma uma lógica de cuidado integral e monitoramento em todas as etapas do envelhecimento, visando uma continuidade das ações, de acordo com o grau de dependência do associado.
Portanto, embora faça parte do modelo a estimativa do percentual de idosos em cada um dos níveis, apenas em uma empresa (Empresa G) foi detectada a preocupação de identificar as prevalências de idosos em cada nível de dependência. Os percentuais expressos no modelo são estimati vas realizadas a partir de dados provenientes de carteiras de empresas, trabal hos internacionais e também com estudos em andamento na UnATI. Deste modo, estima-se que a maioria dos idosos se enquadre no nível 1, com 55\%, sendo que no nível 4 não mais de $5 \%$ da clientela devem estar incluídos.

0 modelo também indica as ações a serem desenvolvidas para cada um dos níveis e o trei- 
namento a ser ministrado para os técnicos que venham a trabal har com este sistema integral de saúde. A preocupação com a capacitação dos profissionais que atuam nos programas das empresas não foi expressa pelos informantes. 0 aprofundamento deste modelo será um dos objetivos de novos estudos, pois além da dificuldade de se operar esta lógica em todas as fases por parte das empresas, também foi identificada a carência de profissionais qualificados para o conjunto das ações.

Um dos maiores desafios dos gestores de operadoras de planos de saúde é medir o Retorno sobre o Investimento (ROI) nos programas de assistência com foco no cuidado integral e monitoramento de pacientes crônicos. Para obter esta estimativa, torna-seimprescindível a elaboração e acompanhamento de indicadores de processo e desfecho destas intervenções. Esteainda éo "calcanhar deAquiles" da maioria das ope radoras que lança o programa de atenção gerenciada de crônicos, mas não implanta a cultura de monitoramento e mensuração dos resultados deste programa.

Outro importante ponto a ser destacado é que, embora haja coerência no discurso sobre a preocupação com os idosos da maioria das empresas, principalmente naquelas três que se destacaram (Empresas K, G e D), o que é ofertado aos seus associados não atende a esta lógica. Observou-se que o discurso do cuidado primário e da promoção de saúde para com os idosos, que faz parte da moderna tecnologia assistencial da geriatria contemporânea, já está incorporado no discurso das empresas de saúde, mas no entanto, quando avaliamos os projetos, detecta-se que o queé ofertado émuito tímido, poisé dirigido para um número reduzido de clientes.

Uma possível explicação para que tal fato ocorra seja porque mesmo com todas as medidas sendo aplicadas, o custo da atenção ao idoso não sereduz. É necessário pontuar queeste custo tende a ser ascendente, pois com o passar dos anos se ampliam às doenças crônicas. Como muitos gestores analisam apenas a planilha de custos e não encontram nenhuma redução significativa, concluem que do ponto de vista financeiro tais projetos não trazem resultado.

Esta análise, no entanto, é incorreta e enganosa, pois se nada for feito os custos se ampliam e com al gumas medidas se conseguiria reduzir o ritmo deste crescimento. Esta informação deve ser mais bem trabalhada no interior das empresas. 0 modelo adotado para a análise propõe melhorar o cuidado e, como resultante desta abordagem mais atual, obter-se um custo mais baixo do que o que tem se praticado. Deve-se frisar, no entanto, que este grupo etário possui múltiplas patologias crônicas e é, por definição, um grupo de alto custo.

Deve-se também considerar que os resultados somente são observados após um período mediano de tempo, talvez um par de anos e para um número expressivo de associados atendidos. Como os programas são desenvolvidos para um número reduzido e a análise de custo é muito imediatista, a resposta esperada é tímida. 0 que seobservou éque as empresas mantêm um pequeno número de associados no programa, apenas para divulgar que possuem tais atividades. Podese mesmo afirmar, pelo reduzido número de clientes nestes programas específicos, que tais atividades têm mais uma função demarketing do que de um cuidado contemporâneo e diferenciado.

Outro fato a ser considerado é que algumas empresas não possuem programas para os idosos, apenas programas para doentes crônicos. A pesar da imensa mai oria dos crônicos ser composta de indivíduos idosos, a lógica do acompanhamento de doenças específicas, apesar de sua validade, não pode ser confundida com o monitoramento permanente do idoso, desde as etapas iniciais da hierarquia do cuidado integral e seu acompanhamento ao longo do processo da maturidade. Como esta atenção não é oferecida, se traduz, em médio prazo, em custos crescentes e cuidado fragmentado, pois se observa apenas as patologias monitoradas no programa.

Um aspecto importante é que nenhuma das empresas possui um programa para idosos com todos os níveis sugeridos pelo modelo, nenhuma mantém ambulatório especializado e nenhuma possui um plano de treinamento para seus técnicos. As próprias empresas reconhecem que o que éofertado épouco epretendem ampliar tais projetos nos próximos anos. A sensação que fica é que não há interesse em qualificar seus profissionais, pois não existe a garantia que o concorrente não irá absorver este profissional treinado, que é muito raro no mercado.

Outra impressão que fica é que, mesmo reconhecendo que possuem ainda programas tímidos, parece que as empresas só iniciarão a sua ampliação se a concorrente assim fizer. 0 temor pela ampliação dos custos prevalece. Assim, criase um círculo vicioso: não se faz, por esperar que a concorrente saia na frentee, pelo fato denão se investir mais nestes programas, não se observa a preocupação com a qualificação de seus técnicos e profissionais para o lidar com o idoso nos vá- 
rios níveis de cuidado. Ao final, modelos específicos para o idoso não são aplicados, por não haver profissionais habilitados e pela empresa se concentrar nos programas já desenvolvidos e conhecidos no mercado, que são o monitoramento das doenças mais prevalentes. Não se deve negar a importância de tais programas, mas eles são apenas parte de um conjunto muito maior de ações para com os idosos.

M erece destaquenegativo o fato deuma grande empresa (Empresa B) não possuir absolutamente nada para o grupo etário dos idosos e os programas existentes serem aplicados apenas para as pessoas ativas, ou seja, o foco é a manutenção da capacidade laboral do indivíduo.

N enhuma empresa expressa a intenção de excluir os idosos de seus quadros; no entanto, fica patente o desejo de não ter a sua carteira ampliada com pessoas mais velhas. 0 tempo despendido em estratégias de bloqueio do ingresso de idosos nas carteiras certamente poderia ser mais bem utilizado no estudo e na elaboração de ações de cuidado integral, como apresentado no modelo utilizado neste trabalho.

\section{Conclusão}

A aceleração no processo de transi ção demográfica eepidemiológica ocasionará um aumento na demanda do atendimento do grupo etário dos idosos. Conformeidentificado no presente estudo, apesar das empresas terem a informação do impacto do ponto de vista de demanda, muito pouco tem sido feito. A proposta que apresentamos no presente trabalho, de um modelo hierarquizado de cuidados, com a perspectiva de englobar todos os níveis de assistência à saúde ${ }^{12}$, e que ten ha a preocupação de captar o cliente certo no momento adequado, além de estratificar os riscos para o cuidado devido, precisa de fato ser implementada. Entendemos que a AN S deva ter uma proposta, com respaldo e fundamentação científica, demodo a poder dial ogar com capacidade de indução e de pressão junto às empresas, como forma de encorajar a inclusão de um modelo mais resolutivo, de melhor qualidade e de custo mais baixo.

Existe nos dias atuais o consenso entre os dirigentes das empresas de saúde da necessidade de implantação de modelos mais contemporâneose resolutivos; no entanto, não se observa nenhum movimento real para que tal discurso se efetive. N otamos que o número de idosos assistidos nos programas para este segmento etário, quando existe, é insignificante e não se busca ampliar. 0 fato relevante é que, pelo menos no discurso, a necessidadedeum ol har especial para com osidosos já foi incorporado, quando no passado nem no discurso o idoso era mencionado.

No entanto, é preciso considerar que o fato da maioria das empresas de planos e seguros de saúde operar por meio de serviços credenciados dificulta a fixação de profissionais e sua educação continuada, que são uma peça-chave para 0 atendimento integral ao idoso.

Por outro lado, os tempos e a lógica dos ciclos de financeirização (curto prazo) que envolvem muitas das prioridades das empresas de planos e seguros de saúde (que atualmente dedicam-se a abrir seus capitais na Bolsa de Valores e optar por investimentos com al tos índices de retorno) nem sempre se coadunam com os requeridos para adoção de inovações de processos e conteúdos assistenciais de longa duração relacionados com a atenção ao idoso. Também podemos observar que existe um enorme abismo entre a prática e o discurso em relação a modelos hierarquizados que priorizem o cuidado integral para com todos os idosos, já que eles possuem patologias crônicas e, neste caso, o modelo de preven ção a ser executado não é a busca da cura, pois na maioria das vezes é impossível, mas a manutenção da capacidadefuncional. Talvez neste aspecto exista um discurso desprovido de um maior embasamento científico. A compreensão de um modelo hierarquizado e integral não está ainda bem assimilada, e as palavras proferidas não representam com exatidão o modelo quenos baseamos para aferir os serviços.

Deste modo, é possível concluir queé necessário elaborar, em maior profundidade, um model o hierarquizado eintegral. 0 modelo utilizado neste estudo éfruto da experiência da UnATI, baseado em modelos mais atuais do mundo. Sua aplicação ficaria mais adequada a partir de um detalhamento maior desta proposta, com uma minuciosa exposição de cada um dos níveis, um aprofundamento na discussão das especificidades do treinamento, da qualificação dos profissionais para cada nível, do novo papel do hospital, e da avaliação econômica deste modelo, entre outros elementos.

A força da preocupação aqui apresentada está, genericamente, na compreensão da heterogeneidade que caracteriza a população idosa, do alto custo dos procedimentos de saúde destinados a esta população, da diversidade dos gastos em subgrupos dessa população e na quantidade insuficiente de especialistas em geriatria. Por outro 
lado, seu valor encontra-se, também, na certeza de que sem uma abordagem preventiva e integral, que associe a reflexão epidemiológica e 0 planejamento sistemático de ações de saúde, não há saída possível para a crise de financiamento e reestruturação do setor.

\section{Colaboradores}

RP Veras e CP Caldas trabal haram na concepção teórica, organização, coleta e análise dos dados, discussão dos resultados, elaboração e redação final do texto. DV Araújo, R Kuschnir eW Mendes participaram na organização e execução da pesquisa, na revisão bibliográfica e na análise e discussão dos resultados.

\section{Referências}

1. Brasil. Ministério da Saúde. Política Nacional de Saúde da Pessoa Idosa. Portaria no 2.529/GM de 19 de outubro de 2006. Institui a Internação Domiciliar no âmbito do SUS. Diário Oficial da U nião 2006; 20 out.

2. Malta $D C$, Jorge $A O$. Breve caracterização da saúde suplementar. In: Ministério da Saúde. Duas faces da mesma moeda: microrregulação e modelos assistenciais na saúde suplementar. Rio de Janeiro: Ministé rio da Saúde/Agência Nacional de Saúde Suplementar; 2005. p. 37-60.

3. Donabedian A. The criteria and standards of quality. v. 2. Ann Harbor, Michigan: Health Administration Press; 1982.

4. Donabedian A. The definition of quality and approach to its assessment. v. 1. Ann Harbor, Michigan: Health Administration Press; 1980.

5. Donabedian A. The methods and findings of quality assessment and monitoring: an illustrated analysis. v. 3. Ann Harbor, Michigan: Health Administration Press; 1985.

6. World Health Organization. Active Ageing: A Policy Framework, 2002 [acessado 2008 Fev 02]. Disponível em: http://www.who.int/ageing
7. Walston J, Hadley EC, Ferrucci L, Guralnik JM, Newman AB, Studenski SA, Ershler WB, Harris T, Fried LP. Research agenda for frailty in older adults: toward a better understanding of physiology and etiology: summary from the American Geriatrics Society/N ational Institute on Aging Research Conference on frailty in older adults. JAGS 2006; 54(6):991-1001.

8. Fried L, Tangen CM, Walston J, Newman AB, Hirsch C, Gottdiener J, Seeman T, Tracy R, Kop WJ, Burke G, McBurnie MA. Frailty in older adults: evidence for a phenotype. J Gerontol A Biol Sci Med Sci. 2001; 56:146- 157.

9. Veras RP, Lourenço RA, M artins CSF, Sanchez MA. Assistência ambulatorial geriátrica: hierarquização da demanda. Rev. Saúde Pública 2005; 39:311-318.

10. Veras RP. Em busca de uma assistência adequada à saúde do idoso: revisão da literatura e aplicação de um instrumento de detecção precoce e de previsibilidade de agravos. Cad Saúde Pública 2003; 19:705-715.

11. Veras RP. Modelos contemporâneos no cuidado à saúde: novos desafios em decorrência da mudança do perfil epidemiológico da população brasileira. Revista da USP 2001; 51:72-85.

12. Leavell S, Clarck EG. M edicina preventiva. São PauIo: McGraw-Hill;1976.

Artigo apresentado em 03/03/2008

Aprovado em 15/04/2008 